\title{
Nonlinear Electroelastic Deformations
}

\author{
A. Dorfmann* and R.W. Ogden ${ }^{\dagger}$ \\ Department of Mathematics, University of Glasgow \\ University Gardens, Glasgow G12 8QW, UK
}

\begin{abstract}
Electro-sensitive (ES) elastomers form a class of smart materials whose mechanical properties can be changed rapidly by the application of an electric field. These materials have attracted considerable interest recently because of their potential for providing relatively cheap and light replacements for mechanical devices, such as actuators, and also for the development of artificial muscles. In this paper we are concerned with a theoretical framework for the analysis of boundary-value problems that underpin the applications of the associated electromechanical interactions. We confine attention to the static situation and first summarize the governing equations for a solid material capable of large electroelastic deformations. The general constitutive laws for the Cauchy stress tensor and the electric field vectors for an isotropic electroelastic material are developed in a compact form following recent work by the authors. The equations are then applied, in the case of an incompressible material, to the solution of a number of representative boundary-value problems. Specifically, we consider the influence of a radial electric field on the azimuthal shear response of a thick-walled circular cylindrical tube, the extension and inflation characteristics of the same tube under either a radial or an axial electric field (or both fields combined), and the effect of a radial field on the deformation of an internally pressurized spherical shell.
\end{abstract}

Mathematics Subject Classification: 74B20, 74F15, 74G05

Keywords: Electroelasticity, Nonlinear elasticity, Electro-active elastomers, Electromechanical interactions

${ }^{*}$ Present address: Department of Civil and Environmental Engineering, Tufts University, 200 College Avenue, Medford, MA 02155, USA

†Communicating author: email rwo@maths.gla.ac.uk 


\section{Introduction}

Electro-sensitive (ES) elastomers are also referred to as electro-active elastomers, and they are a subset of the so-called electro-active polymers (EAPs). These materials deform under the application of an electric field. They have recently attracted growing interest because of their potential for use in actuators, for artificial muscles in robotics and for biomedical applications in prostheses [1]. The mathematical modelling of the properties of such materials, however, is at an early stage of development, partly because of a shortage of sufficient experimental data that can be used for materials characterization.

Even though the engineering applications of ES materials are quite recent, the theoretical foundations of electromagnetic continua within a finite deformation framework date back to the 1950s. We do not attempt to provide a complete account of the relevant background, but instead refer the interested reader to the article by Truesdell and Toupin [16], the lecture notes by Hutter and van de Ven [8], the more recent books by Maugin [11], Eringen and Maugin [5] and Kovetz [10] and references therein. In a recent paper (Dorfmann and Ogden [4]) we have developed a relatively simple but general framework within which the static large deformation electromechanical interaction of ES materials can be analyzed from the phenomenological viewpoint. This involves the construction of a constitutive law for the material expressed through a 'total' energy density function that depends on both the deformation and the electric field (or electric displacement field). The theory was illustrated in [4] by application to two prototype problems, namely the simple shear of a slab (a homogeneous deformation) with the electric field normal to the faces of the slab, and the axial shear of a circular cylindrical tube (a non-homogeneous deformation) in the presence of a radial electric field.

In this paper we build on the formulation of nonlinear electroelasticity in [4] and illustrate the influence of an electric field on the mechanical response of an incompressible isotropic ES elastomer for a number of boundary-value problems.

In Section 2, following Dorfmann and Ogden [4], we summarize briefly the basic electrical and mechanical balance laws for time-independent electric fields. The general constitutive law for an isotropic electroelastic material is then discussed in Section 3. This is based on an amended (or 'total') free energy function for which expressions for the stress and electric field variables assume particularly simple forms. Two alternative formulations are considered. In the first approach the deformation gradient $\mathbf{F}$ and the applied electric field $\mathbf{E}$ are the basic variables. In the second formulation, 
the electric field is replaced by the electric induction vector D. In particular, we provide explicit expressions for the total stress tensor and the electric polarization vector in Eulerian and Lagrangian forms for both compressible and incompressible materials.

In Section 4 we examine two problems for a circular cylindrical tube of incompressible isotropic electroelastic material. First, azimuthal shear in the presence of an initially radial electric field, and second the extension and inflation of a tube with either an axial or a radial electric field (or both). In Section 5 the radial deformation of a spherical shell of the same material in the presence of a radial field is considered. For each problem the general results are illustrated by making a specific simple choice of the energy function. Finally, Section 6 closes the paper with some concluding remarks.

\section{Basic equations}

\section{$2.1 \quad$ Kinematics}

Consider a reference configuration, denoted $\mathcal{B}_{0}$, of the material in which a material particle is labelled by its position vector $\mathbf{X}$. This configuration may or may not be stress free. Let $\mathcal{B}$ denote the corresponding deformed configuration in which the particle $\mathbf{X}$ has position vector $\mathbf{x}$ and the deformation is defined by the mapping $\mathbf{x}=\boldsymbol{\chi}(\mathbf{X})$ for $\mathbf{X} \in \mathcal{B}_{0}$. The deformation gradient tensor, denoted $\mathbf{F}$, is

$$
\mathbf{F}=\operatorname{Grad} \boldsymbol{\chi}
$$

where Grad is the gradient operator in $\mathcal{B}_{0}$. We shall also use the notation $J=\operatorname{det} \mathbf{F}$. By convention we take $J>0$.

\subsection{Electric balance equations}

When the material is deformed, the electric field variables may be defined as Eulerian quantities in the current configuration or as Lagrangian fields in the reference configuration. In this paper we start with the current configuration $\mathcal{B}$ and define the relevant electric field variables as the electric field $\mathbf{E}$, the electric induction $\mathbf{D}$ and the polarization density $\mathbf{P}$. These vectors are related by the standard equation

$$
\mathbf{D}=\varepsilon_{0} \mathbf{E}+\mathbf{P}
$$

where the constant $\varepsilon_{0}$ is the vacuum electric permittivity (see, for example, Kovetz [10]). In vacuo, $\mathbf{P}=\mathbf{0}$ and equation (2) simplifies to $\mathbf{D}=\varepsilon_{0} \mathbf{E}$. In a material $\mathbf{P}$ measures the difference $\mathbf{D}-\varepsilon_{0} \mathbf{E}$ and is a material-dependent property that has to be given by a constitutive equation. 
Here, initially, we take the basic variables to be the electric field $\mathbf{E}$ and the deformation gradient $\mathbf{F}$. Equation (2) then determines the electric induction $\mathbf{D}$ in terms of $\mathbf{F}$ and the field $\mathbf{E}$ when $\mathbf{P}$ is given by a constitutive equation.

For time-independent phenomena and in the absence of magnetic fields, free currents and free electric charges, the vectors $\mathbf{E}$ and $\mathbf{D}$ satisfy the equations

$$
\operatorname{curl} \mathbf{E}=\mathbf{0}, \quad \operatorname{div} \mathbf{D}=0,
$$

obtained by appropriate specialization of Maxwell's equations, where, respectively, curl and div are the curl and divergence operators in $\mathcal{B}$.

The Lagrangian counterparts of the electric field and the electric induction, denoted by $\mathbf{E}_{l}$ and $\mathbf{D}_{l}$ respectively, are given by

$$
\mathbf{E}_{l}=\mathbf{F}^{T} \mathbf{E}, \quad \mathbf{D}_{l}=J \mathbf{F}^{-1} \mathbf{D} .
$$

For details of the derivations of these connections we refer to, for example, Dorfmann and Ogden [4] and references therein. Standard identities ensure that equations (3) are equivalent to

$$
\operatorname{Curl} \mathbf{E}_{l}=\mathbf{0}, \quad \operatorname{Div} \mathbf{D}_{l}=0,
$$

provided $\chi$ is suitably regular, where, respectively, Curl and Div are the curl and divergence operators in $\mathcal{B}_{0}$.

No corresponding pull-back operation for $\mathbf{P}$ arises naturally in a similar way. It is convenient, however, to define a Lagrangian form of $\mathbf{P}$, here denoted $\mathbf{P}_{l}$, analogous to that for $\mathbf{D}$, by

$$
\mathbf{P}_{l}=J \mathbf{F}^{-1} \mathbf{P} .
$$

On using (4) and (6) in (2) we obtain

$$
\mathbf{D}_{l}=\varepsilon_{0} J \mathbf{c}^{-1} \mathbf{E}_{l}+\mathbf{P}_{l},
$$

where $\mathbf{c}^{-1}$ is the inverse of the right Cauchy-Green deformation tensor $\mathbf{c}=$ $\mathbf{F}^{T} \mathbf{F}$.

\subsection{Mechanical balance laws}

Let $\rho_{0}$ and $\rho$ denote the mass densities in the reference and current configurations, respectively. Then, in terms of the notation $J=\operatorname{det} \mathbf{F}$, the conservation of mass equation has the form

$$
J \rho=\rho_{0} .
$$


If the electric body forces are included with the 'total' (Cauchy) stress tensor, denoted $\boldsymbol{\tau}$, the equilibrium equation in the absence of mechanical body forces may be written in the simple form

$$
\operatorname{div} \boldsymbol{\tau}=0,
$$

balance of angular momentum ensuring symmetry of $\boldsymbol{\tau}$, i.e. $\boldsymbol{\tau}^{T}=\boldsymbol{\tau}$.

The counterpart of the nominal stress tensor in elasticity theory, denoted here by $\mathbf{T}$, for the total stress is defined by

$$
\mathbf{T}=J \mathbf{F}^{-1} \boldsymbol{\tau},
$$

and the equilibrium equation (9) may then be written in the alternative form

$$
\operatorname{Div} \mathbf{T}=\mathbf{0} .
$$

\subsection{Boundary conditions}

The electric field $\mathbf{E}$ and the electric induction vector $\mathbf{D}$ satisfy appropriate continuity conditions across any surface within the material or the surface bounding the considered material. In the deformed configuration, in the absence of surface charge, the standard continuity conditions are

$$
\mathbf{n} \cdot[\mathbf{D}]=0, \quad \mathbf{n} \times[\mathbf{E}]=\mathbf{0},
$$

where a square bracket indicates a discontinuity across the surface and $\mathbf{n}$ is normal to the surface. By convention, on the material boundary $\mathbf{n}$ is taken to be the outward pointing normal. These equations may also be given in Lagrangian form (see, for example, [4]), but we omit the details here.

For the mechanical quantities the function $\chi$ has to be continuous across any surface, as has the total traction vector $\boldsymbol{\tau} \mathbf{n}$. The deformation $\mathbf{x}=\boldsymbol{\chi}(\mathbf{X})$ may be prescribed on part of the bounding surface of the body, while the total traction vector on the remaining part of the surface must be continuous. The latter condition is given, in Eulerian form, by

$$
[\boldsymbol{\tau}] \mathbf{n}=\mathbf{0},
$$

where any applied mechanical traction contributes to the traction on the outside. The Maxwell stress there, denoted $\boldsymbol{\tau}_{m}$, must also be accounted for. If the exterior of the body is a vacuum, for example, then $\boldsymbol{\tau}_{m}$ is given by

$$
\boldsymbol{\tau}_{m}=\varepsilon_{0}\left[\mathbf{E} \otimes \mathbf{E}-\frac{1}{2}(\mathbf{E} \cdot \mathbf{E}) \mathbf{I}\right]
$$

where $\mathbf{I}$ is the identity tensor. 


\section{Constitutive Equations}

To complete the formulation of boundary-value problems we need, in addition to the governing equations and boundary conditions, appropriate constitutive laws for the total stress tensor $\boldsymbol{\tau}$ and for the polarization vector $\mathbf{P}$. Following Dorfmann and Ogden [4], we base the construction of constitutive laws on the existence of a free energy function, which may be regarded as a function of the deformation gradient $\mathbf{F}$ and one of the electric field vectors. Here, we take the independent variables initially to be $\mathbf{F}$ and $\mathbf{E}_{l}$, and, in the notation of Dorfmann and Ogden [4], we write the free energy (per unit mass) as

$$
\Phi=\Phi\left(\mathbf{F}, \mathbf{E}_{l}\right) .
$$

It then follows (see, for example, Kovetz [10] and Dorfmann and Ogden [4]) that the total Cauchy stress $\boldsymbol{\tau}$ for a compressible material is given by

$$
\boldsymbol{\tau}=\rho \mathbf{F} \frac{\partial \Phi}{\partial \mathbf{F}}+\boldsymbol{\tau}_{m}
$$

where $\boldsymbol{\tau}_{m}$ is given by (14). The standard requirements of objectivity show that $\Phi$ depends on $\mathbf{F}$ only through $\mathbf{c}=\mathbf{F}^{T} \mathbf{F}$, as in elasticity theory, and symmetry of the first term on the right-hand side of (16) then follows automatically and ensures symmetry of $\boldsymbol{\tau}$. Note, however, that the $\boldsymbol{\tau}_{m}$ inside and outside the material are in general different since $\mathbf{E}$ is different. In the absence of material $\Phi \equiv 0$ and $\boldsymbol{\tau}$ reduces to the Maxwell stress (14).

The expression for the polarization vector in Eulerian form is given in terms of $\Phi$ by

$$
\mathbf{P}=-\rho \mathbf{F} \frac{\partial \Phi}{\partial \mathbf{E}_{l}} .
$$

The corresponding Lagrangian forms of the stress and polarization are obtained on use of equations (10) and (6), respectively. However, rather than giving these explicitly we now make use of a convenient alternative formulation of the constitutive law introduced by Dorfmann and Ogden [4]. This requires the definition of an amended (or 'total') free energy function, denoted $\Omega=\Omega\left(\mathbf{F}, \mathbf{E}_{l}\right)$ and defined per unit reference volume (rather than per unit mass) within the material by

$$
\Omega=\rho_{0} \Phi-\frac{1}{2} \varepsilon_{0} J \mathbf{E}_{l} \cdot\left(\mathbf{c}^{-1} \mathbf{E}_{l}\right) .
$$

(Note that $\mathbf{F}$, and hence $\Omega$, is not defined outside the material.) This allows us to write the total stress tensors $\boldsymbol{\tau}$ and $\mathbf{T}$ in the compact forms

$$
\boldsymbol{\tau}=J^{-1} \mathbf{F} \frac{\partial \Omega}{\partial \mathbf{F}}, \quad \mathbf{T}=\frac{\partial \Omega}{\partial \mathbf{F}} .
$$


While it is the polarization that is given by (17), it is now the electric displacement that is given directly in terms of $\Omega$. In Eulerian form the polarization and electric displacement are given by

$$
\mathbf{P}=\mathbf{D}-\varepsilon_{0} \mathbf{E}, \quad \mathbf{D}=-J^{-1} \mathbf{F} \frac{\partial \Omega}{\partial \mathbf{E}_{l}}
$$

and their Lagrangian counterparts by

$$
\mathbf{P}_{l}=\mathbf{D}_{l}-\varepsilon_{0} J \mathbf{c}^{-1} \mathbf{E}_{l}, \quad \mathbf{D}_{l}=-\frac{\partial \Omega}{\partial \mathbf{E}_{l}} .
$$

The expressions listed above for the stresses require modification in the case of incompressible materials, which are subject to the constraint

$$
J=\operatorname{det} \mathbf{F} \equiv 1
$$

so that (18) becomes

$$
\Omega=\rho_{0} \Phi-\frac{1}{2} \varepsilon_{0} \mathbf{E}_{l} \cdot\left(\mathbf{c}^{-1} \mathbf{E}_{l}\right)
$$

The total stress tensors (19) are replaced by

$$
\boldsymbol{\tau}=\mathbf{F} \frac{\partial \Omega}{\partial \mathbf{F}}-p \mathbf{I}, \quad \mathbf{T}=\frac{\partial \Omega}{\partial \mathbf{F}}-p \mathbf{F}^{-1},
$$

where $p$ is a Lagrange multiplier associated with the constraint (22). The expressions for the electric induction and the polarization fields given in Eulerian and Lagrangian forms by equations (20) and (21), respectively, are unchanged but subject to (22).

\section{$3.1 \quad$ Isotropy}

Application of an electric field to an isotropic ES elastomer introduces, locally, a preferred direction analogous to that arising for transversely isotropic elastic solids. Following the analysis of such materials given in Spencer [14] and Ogden [13], for example, we define an isotropic ES material as one for which $\Omega$ is an isotropic function of $\mathbf{c}$ and $\mathbf{E}_{l} \otimes \mathbf{E}_{l}$. The form of $\Omega$ is then reduced to dependence on the six independent invariants, denoted $I_{1}, I_{2}, \ldots, I_{6}$, of $\mathbf{c}$ and $\mathbf{E}_{l} \otimes \mathbf{E}_{l}$. For a compressible material, we choose the standard principal invariants of $\mathbf{c}$, namely

$$
I_{1}=\operatorname{tr} \mathbf{c}, \quad I_{2}=\frac{1}{2}\left[(\operatorname{tr} \mathbf{c})^{2}-\operatorname{tr}\left(\mathbf{c}^{2}\right)\right], \quad I_{3}=\operatorname{det} \mathbf{c}=J^{2},
$$


while for the invariants depending on $\mathbf{E}_{l}$ we set

$$
I_{4}=\left|\mathbf{E}_{l}\right|^{2}, \quad I_{5}=\mathbf{E}_{l} \cdot\left(\mathbf{c}^{-1} \mathbf{E}_{l}\right), \quad I_{6}=\mathbf{E}_{l} \cdot\left(\mathbf{c}^{-2} \mathbf{E}_{l}\right),
$$

where $\operatorname{tr}$ is the trace of a second-order tensor. Note that $I_{5}$ and $I_{6}$ can also be written, respectively, as $\mathbf{E} \cdot \mathbf{E}$ and $\mathbf{E} \cdot\left(\mathbf{b}^{-1} \mathbf{E}\right)$, where $\mathbf{b}=\mathbf{F} \mathbf{F}^{T}$ is the left Cauchy-Green deformation tensor. The choice (26) is not, of course, unique and one could, for example, replace $\mathbf{c}^{-1}$ by $\mathbf{c}$ in $I_{5}$ and $I_{6}$. For incompressible materials the invariant $I_{3} \equiv 1$ is omitted.

For an incompressible isotropic material, therefore, $\Omega=\Omega\left(I_{1}, I_{2}, I_{4}, I_{5}, I_{6}\right)$, and the explicit forms of the total stress tensor $\boldsymbol{\tau}$ and the electric induction vector $\mathbf{D}$ are

$$
\begin{aligned}
\boldsymbol{\tau}= & 2 \Omega_{1} \mathbf{b}+2 \Omega_{2}\left(I_{1} \mathbf{b}-\mathbf{b}^{2}\right)-p \mathbf{I} \\
& -2 \Omega_{5} \mathbf{E} \otimes \mathbf{E}-2 \Omega_{6}\left(\mathbf{b}^{-1} \mathbf{E} \otimes \mathbf{E}+\mathbf{E} \otimes \mathbf{b}^{-1} \mathbf{E}\right), \\
\mathbf{D}= & -2\left(\Omega_{4} \mathbf{b} \mathbf{E}+\Omega_{5} \mathbf{E}+\Omega_{6} \mathbf{b}^{-1} \mathbf{E}\right),
\end{aligned}
$$

where the subscripts $1,2,4,5,6$ on $\Omega$ signify partial differentiation with respect to $I_{1}, I_{2}, I_{4}, I_{5}, I_{6}$, respectively, and wherein the left Cauchy-Green deformation tensor $\mathbf{b}$ is used.

\subsection{Change of independent variables}

In the solution of boundary-value problems involving non-uniform fields, it may in some circumstances be more convenient to select $\mathbf{D}_{l}$ as the independent electric variable instead of $\mathbf{E}_{l}$. This can be done by defining an energy function $\Omega^{*}=\Omega^{*}\left(\mathbf{F}, \mathbf{D}_{l}\right)$, complementary to $\Omega$, via the Legendre-type transform

$$
\Omega^{*}\left(\mathbf{F}, \mathbf{D}_{l}\right)=\Omega\left(\mathbf{F}, \mathbf{E}_{l}\right)+\mathbf{D}_{l} \cdot \mathbf{E}_{l} .
$$

The total stress tensor and the electric field in Lagrangian form, for compressible materials, are then simply

$$
\mathbf{T}=\frac{\partial \Omega^{*}}{\partial \mathbf{F}}, \quad \mathbf{E}_{l}=\frac{\partial \Omega^{*}}{\partial \mathbf{D}_{l}}
$$

and the polarization is still given by $(21)_{1}$, but now with $\mathbf{D}_{l}$ as the independent variable and $\mathbf{E}_{l}$ given by $(30)_{2}$.

For an isotropic material, $\Omega^{*}$ depends on the invariants $I_{1}, I_{2}, I_{3}$ defined in (25) and on three invariants based on $\mathbf{D}_{l}$, for which we use the notation $K_{4}, K_{5}, K_{6}$. We choose to define these as

$$
K_{4}=\mathbf{D}_{l} \cdot \mathbf{D}_{l}, \quad K_{5}=\mathbf{D}_{l} \cdot\left(\mathbf{c D}_{l}\right), \quad K_{6}=\mathbf{D}_{l} \cdot\left(\mathbf{c}^{2} \mathbf{D}_{l}\right)
$$


For an incompressible material, the Eulerian form of the total stress $\boldsymbol{\tau}$ and the electric field $\mathbf{E}$ based on $\Omega^{*}$ have the explicit forms

$$
\begin{aligned}
\boldsymbol{\tau}= & 2 \Omega_{1}^{*} \mathbf{b}+2 \Omega_{2}^{*}\left(I_{1} \mathbf{b}-\mathbf{b}^{2}\right)-p \mathbf{I} \\
& +2 \Omega_{5}^{*} \mathbf{D} \otimes \mathbf{D}+2 \Omega_{6}^{*}(\mathbf{D} \otimes \mathbf{b} \mathbf{D}+\mathbf{b} \mathbf{D} \otimes \mathbf{D}), \\
\mathbf{E}= & 2\left(\Omega_{4}^{*} \mathbf{b}^{-1} \mathbf{D}+\Omega_{5}^{*} \mathbf{D}+\Omega_{6}^{*} \mathbf{b} \mathbf{D}\right) .
\end{aligned}
$$

The polarization is again given by $(20)_{1}$ with $\mathbf{D}=\mathbf{F D}_{l}$. Here, we define $\Omega_{i}^{*}$ to be $\partial \Omega^{*} / \partial I_{i}$ for $i=1,2$ and $\partial \Omega^{*} / \partial K_{i}$ for $i=4,5,6$.

\subsection{The reference configuration}

If the material is not subject to any mechanical boundary tractions or mechanical body forces then, in general, application of an electric field will induce the material to deform, a phenomenon known as electrostriction. Let the resulting configuration be taken as the reference configuration, which we now denote by $\mathcal{B}_{r}$ to distinguish it from $\mathcal{B}_{0}$. These two reference configurations can be taken to coincide if appropriate loads are applied to the body, which will result in a (residual) stress distribution throughout the material. In such a case we denote the values of $\boldsymbol{\tau}, \mathbf{E}, \mathbf{D}$ and $\mathbf{P}$ in this configuration by $\boldsymbol{\tau}_{0}, \mathbf{E}_{0}, \mathbf{D}_{0}$ and $\mathbf{P}_{0}$, respectively. Again we focus on incompressible materials. With $I_{3} \equiv 1$ and $\mathbf{F}=\mathbf{I}$ the invariants (25), (26) and (31) reduce to

$$
I_{1}=I_{2}=3, \quad I_{4}=I_{5}=I_{6}=\mathbf{E}_{0} \cdot \mathbf{E}_{0}, \quad K_{4}=K_{5}=K_{6}=\mathbf{D}_{0} \cdot \mathbf{D}_{0} .
$$

Then, in terms of $\Omega$ and $\Omega^{*}$ the expressions for the total stress tensor $\boldsymbol{\tau}_{0}$ simplify to

$$
\boldsymbol{\tau}_{0}=\left[2\left(\Omega_{1}+2 \Omega_{2}\right)-p\right] \mathbf{I}-2\left(\Omega_{5}+2 \Omega_{6}\right) \mathbf{E}_{0} \otimes \mathbf{E}_{0},
$$

and

$$
\boldsymbol{\tau}_{0}=\left[2\left(\Omega_{1}^{*}+2 \Omega_{2}^{*}\right)-p\right] \mathbf{I}+2\left(\Omega_{5}^{*}+2 \Omega_{6}^{*}\right) \mathbf{D}_{0} \otimes \mathbf{D}_{0},
$$

respectively, with $\Omega_{i}$ and $\Omega_{i}^{*}$ evaluated for the appropriate subset of invariants (34).

The corresponding expressions for the electric field vectors may be simplified by defining $\Omega_{0}\left(I_{4}\right) \equiv \Omega\left(3,3, I_{4}, I_{4}, I_{4}\right)$ and $\Omega_{0}^{*}\left(K_{4}\right) \equiv \Omega^{*}\left(3,3, K_{4}, K_{4}, K_{4}\right)$. Then, we obtain the specializations of $\mathbf{D}$ in (28) and $\mathbf{P}$ as

$$
\mathbf{D}_{0}=-2 \Omega_{0}^{\prime}\left(I_{4}\right) \mathbf{E}_{0}, \quad \mathbf{P}_{0}=\mathbf{D}_{0}-\varepsilon_{0} \mathbf{E}_{0},
$$

where the prime signifies differentiation with respect to $I_{4}$. 
Similarly, for $\mathbf{E}$ in (33) and $\mathbf{P}$ the specializations are

$$
\mathbf{E}_{0}=2 \Omega_{0}^{* \prime}\left(K_{4}\right) \mathbf{D}_{0}, \quad \mathbf{P}_{0}=\mathbf{D}_{0}-\varepsilon_{0} \mathbf{E}_{0},
$$

with the prime signifying differentiation with respect to $K_{4}$.

In this configuration $\mathbf{E}_{0}, \mathbf{D}_{0}$ and $\boldsymbol{\tau}_{0}$ must satisfy the equations

$$
\operatorname{Curl} \mathbf{E}_{0}=\mathbf{0}, \quad \operatorname{Div} \mathbf{D}_{0}=0, \quad \operatorname{Div} \boldsymbol{\tau}_{0}=\mathbf{0} .
$$

\section{Problems with cylindrical symmetry}

We consider an incompressible isotropic ES material in the absence of mechanical body forces and subject to an (in general non-uniform) electric field. The analysis is based on use of either the energy function $\Omega$, regarded as a function of the invariants $I_{1}, I_{2}, I_{4}, I_{5}, I_{6}$, or $\Omega^{*}$, as a function of $I_{1}, I_{2}, K_{4}, K_{5}, K_{6}$. We work in terms of the Eulerian versions of the field equations, which are collected together here as

$$
\operatorname{div} \boldsymbol{\tau}=\mathbf{0}, \quad \operatorname{curl} \mathbf{E}=\mathbf{0}, \quad \operatorname{div} \mathbf{D}=0 .
$$

For the formulation based on $\Omega$ the explicit expressions for the total stress tensor $\boldsymbol{\tau}$ and the electric displacement $\mathbf{D}$ are as given in equations (27) and (28), with $\mathbf{E}=\mathbf{F}^{-T} \mathbf{E}_{l}$, while for the formulation in terms of $\Omega^{*}, \boldsymbol{\tau}$ is given by (32) and the electric field $\mathbf{E}$ by (33), with $\mathbf{D}=\mathbf{F D}_{l}$.

We now specialize these equations for the geometry of a thick-walled circular cylindrical tube for which cylindrical symmetry is maintained during deformation. We work in terms of cylindrical polar coordinates. In the reference configuration these are denoted by $(R, \Theta, Z)$ and in the deformed configuration by $(r, \theta, z)$. We begin by considering the reference configuration, in which equations $(39)_{1,2}$ take the explicit forms

$$
\frac{1}{R} \frac{\partial E_{0 Z}}{\partial \Theta}-\frac{\partial E_{0 \Theta}}{\partial Z}=0, \frac{\partial E_{0 R}}{\partial Z}-\frac{\partial E_{0 Z}}{\partial R}=0, \frac{1}{R} \frac{\partial\left(R E_{0 \Theta}\right)}{\partial R}-\frac{1}{R} \frac{\partial E_{0 R}}{\partial \Theta}=0,
$$

and

$$
\frac{\partial D_{0 R}}{\partial R}+\frac{1}{R} D_{0 R}+\frac{1}{R} \frac{\partial D_{0 \Theta}}{\partial \Theta}+\frac{\partial D_{0 Z}}{\partial Z}=0,
$$

where $\left(E_{0 R}, E_{0 \Theta}, E_{0 Z}\right)$ are the components of the applied electric field $\mathbf{E}_{0}$ and $\left(D_{0 R}, D_{0 \Theta}, D_{0 Z}\right)$ those of the electric induction $\mathbf{D}_{0}$.

Since we are assuming cylindrical symmetry there is no dependence on either $\Theta$ or $Z$ and the above equations may therefore be integrated to give

$$
R D_{0 R}=\text { constant }, \quad R E_{0 \Theta}=\text { constant }, \quad E_{0 Z}=\text { constant } .
$$


The first thing to note, in the case of the component $E_{0 \Theta}$, is that the associated electrostatic potential has the form $c \Theta+d$, where $c$ and $d$ are constants. This function is not single valued, and hence, for a complete tube $(0 \leq \Theta \leq 2 \pi)$, must be discounted. Thus, we take $E_{0 \Theta}=0$ and in the following consider only radial and axial electric fields.

A general comment is in order concerning the considered geometry. Strictly, the analysis presented below applies only for a tube of infinite length since the boundary conditions for $\mathbf{E}$ and $\mathbf{D}$ on the ends of a tube of finite length are not in general compatible with those on the lateral surfaces of the tube. Thus, end effects will be present. However, if the tube is of sufficient length compared with its outer radius then the influence of such effects can be neglected throughout most of the tube length.

\subsection{Azimuthal shear}

The cross-sectional geometry of the tube in the reference configuration is specified by

$$
A \leq R \leq B, \quad 0 \leq \Theta \leq 2 \pi,
$$

where $A$ and $B$ are the inner and outer radii, respectively. A (pure) azimuthal shear deformation is defined by

$$
r=R, \quad \theta=\Theta+\phi(R), \quad z=Z,
$$

where $\phi$ is a function of $R$ that has to be determined by solution of the governing equations and application of the boundary conditions. For details of this deformation for a purely elastic material, we refer to Jiang and Ogden [9], for example, and references therein.

Referred to the two sets of cylindrical polar coordinate axes, the component matrix of the deformation gradient $\mathbf{F}$, denoted $F$, is given by

$$
\mathrm{F}=\left(\begin{array}{ccc}
1 & 0 & 0 \\
r \phi^{\prime}(r) & 1 & 0 \\
0 & 0 & 1
\end{array}\right)
$$

where, since $r=R, \phi$ is now being treated as a function of $r$. For notational convenience, we write $\gamma=r \phi^{\prime}(r)$, with the prime indicating differentiation with respect to $r$, and note that the deformation is locally a simple shear with amount of shear $\gamma$. The corresponding matrices for $\mathbf{b}$ and $\mathbf{c}$, written $\mathbf{b}$ and c, are

$$
\mathbf{b}=\left(\begin{array}{ccc}
1 & \gamma & 0 \\
\gamma & 1+\gamma^{2} & 0 \\
0 & 0 & 1
\end{array}\right), \quad \mathbf{c}=\left(\begin{array}{ccc}
1+\gamma^{2} & \gamma & 0 \\
\gamma & 1 & 0 \\
0 & 0 & 1
\end{array}\right)
$$


and the associated principal invariants are, from (25),

$$
I_{1}=I_{2}=3+\gamma^{2}, \quad I_{3} \equiv 1 .
$$

For the considered circular symmetry the equilibrium equation $(40)_{1}$ reduces to the radial and azimuthal component equations

$$
r \frac{\mathrm{d} \tau_{r r}}{\mathrm{~d} r}=\tau_{\theta \theta}-\tau_{r r}, \quad \frac{d}{d r}\left(r^{2} \tau_{r \theta}\right)=0
$$

\subsubsection{Electric (displacement) field}

We work in terms of the energy function $\Omega^{*}$ and identify the Lagrangian field $\mathbf{D}_{l}$ with the initial field $\mathbf{D}_{0}$ in the reference configuration $\mathcal{B}_{0}$. (Because of the incompressibility and the form of the considered deformation, there is no electrostriction associated with $\mathbf{D}_{0}$ and $\mathcal{B}_{r}=\mathcal{B}_{0}$.) It follows that the field $\mathbf{D}=\mathbf{F D}_{0}$ in the deformed configuration $\mathcal{B}$ automatically satisfies the field equation $\operatorname{div} \mathbf{D}=0$. For definiteness we take $\mathbf{D}_{0}$ to be purely radial with radial component $D_{0 R}$. The invariants $K_{4}, K_{5}, K_{6}$ are then calculated from (31) and (47) $)_{2}$ as

$$
K_{4}=D_{0 R}^{2}, \quad K_{5}=\left(1+\gamma^{2}\right) K_{4}, \quad K_{6}=\left(1+3 \gamma^{2}+\gamma^{4}\right) K_{4},
$$

which are functions of the radius $r$.

The components of $\mathbf{D}$ in the deformed configuration are calculated from the component form of $\mathbf{D}=\mathbf{F} \mathbf{D}_{0}$ as

$$
D_{r}=D_{0 R}, \quad D_{\theta}=\gamma D_{0 R}, \quad D_{z}=0,
$$

while the components of the electric field $\mathbf{E}$ are given by (33) as

$$
E_{r}=2\left[\Omega_{4}^{*}+\Omega_{5}^{*}+\left(1+\gamma^{2}\right) \Omega_{6}^{*}\right] D_{0 R}, \quad E_{\theta}=2 \gamma\left[\Omega_{5}^{*}+\left(2+\gamma^{2}\right) \Omega_{6}^{*}\right] D_{0 R},
$$

with the axial component $E_{z}=0$. Note, in particular, that $\mathbf{F}^{-T} \mathbf{E}_{0}$ is not in general the same as $\mathbf{E}$, i.e. $\mathbf{E}_{l} \neq \mathbf{E}_{0}$, and hence $\mathbf{E}$ does not automatically satisfy the field equation curl $\mathbf{E}=\mathbf{0}$. Because of the cylindrical symmetry, $E_{\theta}$ must vanish for the same reason as $E_{0 \theta}$ vanishes, and, hence, we must have

$$
\Omega_{5}^{*}+\left(2+\gamma^{2}\right) \Omega_{6}^{*}=0 .
$$

This condition also arises in the helical shear problem, which was discussed briefly by Bustamante and Ogden [2] in the context of universal relations.

In general, for a given form of $\Omega^{*}$, equation (53) will not be satisfied. However, it can be satisfied for particular classes of energy function $\Omega^{*}$. 
Bearing in mind that, for the considered deformation, we have $I_{2}=I_{1}=$ $3+\gamma^{2}$, one such class is given by

$$
\Omega^{*}=G\left(I_{1}, I_{2}, K_{4}\right)+H\left(I_{1}, I_{2}, K\right),
$$

where $K=K_{6}-\left(I_{1}-1\right) K_{5}$ and $G$ and $H$ are 'arbitrary', but suitably well-behaved, functions.

Given that the above restrictions are satisfied, and hence $E_{\theta}=0$, the components of the polarization are given by

$$
P_{r}=D_{0 R}-2 \varepsilon_{0}\left(\Omega_{4}^{*}-\Omega_{6}^{*}\right) D_{0 R}, \quad P_{\theta}=\gamma D_{0 R}, \quad P_{z}=0,
$$

use having been made of (53) in the expression for $P_{r}$.

Restrictions, such as (53), on the energy function arise naturally because of the assumptions on the form of geometry, deformation and electric (or electric displacement) field, as demonstrated previously by Dorfmann and Ogden [4] for electroelastic problems. Similar restrictions arise in magnetoelastic problems, as discussed, for example, by Dorfmann and Ogden [3]. This is a consequence of the semi-inverse approach adopted.

\subsubsection{Stress components and equilibrium}

Turning next to the stress components, we calculate the (non-zero) components of $\boldsymbol{\tau}$ from equation (32) as

$$
\begin{aligned}
\tau_{r r} & =-p+2\left(\Omega_{1}^{*}+2 \Omega_{2}^{*}\right)+2\left[\Omega_{5}^{*}+2\left(1+\gamma^{2}\right) \Omega_{6}^{*}\right] K_{4}, \\
\tau_{\theta \theta} & =-p+2\left(\Omega_{1}^{*}+2 \Omega_{2}^{*}\right)+2 \gamma^{2}\left(\Omega_{1}^{*}+\Omega_{2}^{*}\right)+2 \gamma^{2}\left[\Omega_{5}^{*}+2\left(2+\gamma^{2}\right) \Omega_{6}^{*}\right] K_{4}, \\
\tau_{z z} & =-p+2\left[\Omega_{1}^{*}+\left(2+\gamma^{2}\right) \Omega_{2}^{*}\right] \\
\tau_{r \theta} & =2 \gamma\left(\Omega_{1}^{*}+\Omega_{2}^{*}\right)+2 \gamma\left[\Omega_{5}^{*}+\left(3+2 \gamma^{2}\right) \Omega_{6}^{*}\right] K_{4} .
\end{aligned}
$$

In view of (48) and (50) the invariants can all be expressed in terms of the independent quantities $\gamma$ and $K_{4}$. It is therefore convenient to define a reduced form $\omega^{*}$ of the energy function by

$$
\omega^{*}\left(\gamma, K_{4}\right)=\Omega^{*}\left(I_{1}, I_{2}, K_{4}, K_{5}, K_{6}\right),
$$

where the right-hand side is evaluated for (48) and (50). The expressions for $\tau_{r \theta}$ and $E_{r}$ then simplify to

$$
\tau_{r \theta}=\omega_{\gamma}^{*}, \quad E_{r}=2 \omega_{4}^{*} D_{0 R},
$$

where $\omega_{\gamma}^{*}=\partial \omega^{*} / \partial \gamma$ and $\omega_{4}^{*}=\partial \omega^{*} / \partial K_{4}$. 
The connection

$$
\tau_{\theta \theta}-\tau_{r r}-\gamma \tau_{r \theta}=-2 \gamma^{2} K_{4} \Omega_{6}^{*}
$$

can also be shown to hold. Note that the right-hand side of (62) vanishes if $\Omega^{*}$ is independent of $K_{6}$. Then, however, by (53), $\Omega^{*}$ must also be independent of $K_{5}$. In this case equation (62) yields a 'universal relation' between the components of stress, i.e. a relation independent of the form of energy function within the restricted class of functions $\Omega^{*}=\Omega^{*}\left(I_{1}, I_{2}, K_{4}\right)$, identical to that arising in the corresponding purely elastic problem. Of course, if $K_{4}=0$ the elastic result is recovered.

Combination of the integration of equation $(49)_{2}$ with $(61)_{1}$ leads to

$$
\omega_{\gamma}^{*}=\frac{\tau_{\theta} b^{2}}{r^{2}},
$$

where $\tau_{\theta}$ is the value of the shear stress $\tau_{r \theta}$ on the boundary $r=b$. For a given $\Omega^{*}$, and hence $\omega^{*}$, and for a known electric field component $D_{0 R}$, equation (63) can in principle be solved for $\gamma=r \phi^{\prime}(r)$, and then the deformation function $\phi(r)$ is obtained by integration.

For the azimuthal shear problem considered here, we take the boundary conditions to be

$$
\phi(a)=0, \quad \phi(b)=\psi,
$$

which correspond to the inner boundary being held fixed and the outer boundary rotated by a given angle $\psi$.

Equation $(49)_{1}$ is not used here since, in conjunction with the Maxwell stress on the tube boundaries $r=a, b$, it merely determines the radial traction on the boundaries, which are subject to the placement boundary conditions (64).

\subsubsection{Illustration}

To illustrate the effect of an electric field on the response of the considered material under large azimuthal deformations, we consider the reduced energy function

$$
\omega^{*}\left(\gamma, K_{4}\right)=\frac{\mu^{*}\left(K_{4}\right)}{k}\left[\left(\frac{2+\gamma^{2}}{2}\right)^{k}-1\right]+\nu^{*}\left(K_{4}\right),
$$

where $k$ is a constant and $\mu^{*}$ is the shear modulus of the material in the undeformed configuration $(\gamma=0)$ and depends on the electric field through the invariant $K_{4}$, as does the function $\nu^{*}$. The latter function describes the energy (per unit volume) in the material associated with the electric field when $\gamma=0$ and must satisfy the condition $\nu^{*}(0)=0$. 
From $(61)_{1}$ we calculate the associated shear stress

$$
\tau_{r \theta}=\mu^{*}\left(K_{4}\right) \gamma\left(\frac{2+\gamma^{2}}{2}\right)^{k-1}
$$

and we note, in particular, that this does not depend on the function $\nu^{*}\left(K_{4}\right)$. Also, from $(61)_{2}$, we obtain

$$
E_{r}=2 \frac{\mu^{* \prime}\left(K_{4}\right)}{k}\left[\left(\frac{2+\gamma^{2}}{2}\right)^{k}-1\right] D_{0 R}+2 \nu^{* \prime}\left(K_{4}\right) D_{0 R},
$$

which reduces to

$$
E_{0 R}=2 \nu^{* \prime}\left(K_{4}\right) D_{0 R}
$$

when $\gamma=0$. The corresponding polarization component is, by $(38)_{2}$,

$$
P_{0 R}=\left[1-2 \varepsilon_{0} \nu^{* \prime}\left(K_{4}\right)\right] D_{0 R},
$$

which shows that the function $\nu^{*}\left(K_{4}\right)$, through its derivative, is a measure of the polarization in the material.

In the absence of an electric field the energy function reduces to the strain-energy function of an elastic material, given by

$$
\omega^{*}(\gamma, 0)=\frac{\mu^{*}(0)}{k}\left[\left(\frac{2+\gamma^{2}}{2}\right)^{k}-1\right],
$$

for the considered deformation. Note that $\mu^{*}(0)$ corresponds to the shear modulus of the elastic material in the reference configuration.

The strain-energy function (70) is one of the classes of energy functions considered by Jiang and Ogden [9]. Thus, equation (65) can be seen as a natural extension of this class to the electroelastic situation. More generally, we can consider (65) as being derived from an $\Omega^{*}=\Omega^{*}\left(I_{1}, K_{4}\right)$ given by

$$
\Omega^{*}\left(I_{1}, K_{4}\right)=\frac{\mu^{*}\left(K_{4}\right)}{k}\left[\left(\frac{I_{1}-1}{2}\right)^{k}-1\right]+\nu^{*}\left(K_{4}\right),
$$

although this is not the only possible form of $\Omega^{*}$ that reduces to the particular form of $\omega^{*}$ given by (65). Note that the energy function (71) satisfies the condition (53). As discussed in Jiang and Ogden [9], for the shear stress to be a monotonic increasing function of $\gamma$ we must have $k \geq 1 / 2$.

For the energy function (65) the azimuthal shear response equation (63) gives

$$
\mu^{*}\left(K_{4}\right) \gamma\left(\frac{2+\gamma^{2}}{2}\right)^{k-1}=\frac{\tau_{\theta} b^{2}}{r^{2}}
$$


and we recall that $\gamma=r \phi^{\prime}(r)$.

For definiteness and for purposes of numerical calculation, we now take $k=3 / 4$ and $\mu^{*}\left(K_{4}\right)$ to depend linearly on $K_{4}$ in the form

$$
\mu^{*}\left(K_{4}\right)=\mu^{*}(0)+\mu_{1}^{*} K_{4},
$$

where $\mu_{1}^{*}$ is a positive material constant. We recall that $K_{4}=D_{0 R}^{2}$ and that $D_{0 R}=c / r$, where $c$ is a constant quantifying the magnitude of the applied electric field. It will be convenient in what follows to define the dimensionless quantities

$$
\tau=\tau_{\theta} / \mu^{*}(0), \quad \bar{r}=r / b, \quad e=\mu_{1}^{*} c^{2} / \mu^{*}(0) b^{2},
$$

and we note, in particular, that $e$ reflects the material properties through the ratio $\mu_{1}^{*} / \mu^{*}(0)$ and the magnitude of the electric field through $c$.

Equation (72) now reduces to

$$
\gamma\left(1+\frac{1}{2} \gamma^{2}\right)^{-1 / 4}=x
$$

where $x$ is defined as

$$
x=\frac{\tau}{\bar{r}^{2}+e} .
$$

This allows an explicit unique positive solution to be obtained for $\gamma$ in the form

$$
\gamma=\frac{1}{2} x\left[x^{2}+\left(x^{4}+16\right)^{1 / 2}\right]^{1 / 2} .
$$

Next, using the connection $\gamma=r \phi^{\prime}(r)$ and the boundary conditions (64), we obtain the integral

$$
\psi=\phi(b)=\frac{1}{2} \int_{a}^{b} x\left[x^{2}+\left(x^{4}+16\right)^{1 / 2}\right]^{1 / 2} \frac{\mathrm{d} r}{r} .
$$

On changing the variable of integration to $x$ this becomes

$$
\psi=\frac{\tau}{4} \int_{\tau /(1+e)}^{\tau /(\eta+e)}\left[x^{2}+\left(x^{4}+16\right)^{1 / 2}\right]^{1 / 2} \frac{\mathrm{d} x}{\tau-e x},
$$

where $\eta=a^{2} / b^{2}$.

Equation (79) gives $\psi$ as a function of $\tau$ when the values of $\eta$ and $e$ are specified. In Figure 1, based on this equation, we plot $\tau$ as a function of $\psi$ for the case of no electric field $(e=0)$ and for the two values $0.2,0.4$ of $e$. We have set $\eta=0.5(b=a \sqrt{2})$, which represents a moderate value of the tube thickness. Note that the integral (79) can be evaluated in closed 


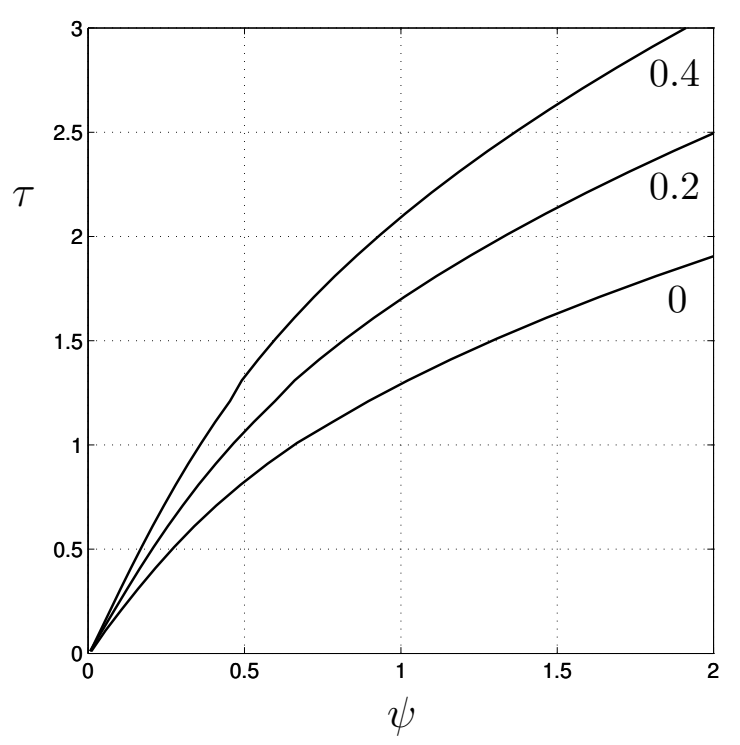

Figure 1: Plot of the dimensionless shear stress $\tau$ on $r=b$ against the angle of rotation $\psi$ based on equation (79) for $e=0,0.2,0.4$ and $\eta=0.5$.

form for $e=0$ (see Jiang and Ogden [9] for the result). Figure 1 shows how the presence of an electric field stiffens the mechanical shear response of the material, which reflects the choice of the specific form of the function $\mu^{*}\left(K_{4}\right)$ in $(73)$.

Finally in this section we remark that the formulation based on $\Omega$ rather than $\Omega^{*}$ can equally well be used for this problem, with rather similar results, except that the restrictions on $\Omega$ analogous to (53) are somewhat different. We omit the details here and refer to the discussion in Bustamante and Ogden [2] with reference to helical shear. A discussion on the consequences of using formulations based on $\Omega$ and $\Omega^{*}$ for the corresponding magnetoelastic situation is provided by Dorfmann and Ogden [3]. A parallel analysis can be conducted for an axial electric field instead of a radial field, but the general pattern of the results is similar to that presented here so is not given separately.

\subsection{Extension and inflation of a tube}

In this section we apply the theory of ES materials to a circular cylindrical tube with reference configuration in cylindrical polar coordinates $(R, \Theta, Z)$ given again by (44). The deformation consists of a combined uniform axial extension (or contraction) and radial inflation (or deflation), with cylindrical 
symmetry maintained. All variables again depend only on the radius $r$. The cross-section of the deformed configuration is described in the cylindrical polar coordinates $(r, \theta, z)$ by the equations

$$
a \leq r \leq b, \quad 0 \leq \theta \leq 2 \pi
$$

Again we are considering an incompressible material and the deformation is therefore expressed in the standard form

$$
r^{2}=a^{2}+\lambda_{z}^{-1}\left(R^{2}-A^{2}\right), \quad \theta=\Theta, \quad z=\lambda_{z} Z,
$$

where $\lambda_{z}$ is the axial stretch, which is independent of $r$. The component matrix of the deformation gradient $\mathbf{F}$ is diagonal with respect to the two sets of cylindrical polar coordinate axes and its $(r, \theta, z)$ normal components are $\left(\lambda^{-1} \lambda_{z}^{-1}, \lambda, \lambda_{z}\right)$, respectively, where $\lambda=r / R$ is the azimuthal stretch and $\lambda^{-1} \lambda_{z}^{-1}$ the radial stretch.

Because of the incompressibility condition, this deformation may be described by two independent stretches, and for this purpose we use $\lambda$ and $\lambda_{z}$. The two independent invariants $I_{1}$ and $I_{2}$ defined in $(25)_{1,2}$ have the forms

$$
I_{1}=\lambda^{-2} \lambda_{z}^{-2}+\lambda^{2}+\lambda_{z}^{2}, \quad I_{2}=\lambda^{2} \lambda_{z}^{2}+\lambda^{-2}+\lambda_{z}^{-2}
$$

in terms of the stretches.

For this problem we use the formulation based on $\Omega$ with the electric field $\mathbf{E}_{l}$ as the independent variable. In the azimuthal problem we considered an initial electric field in the (undeformed) reference configuration and this was possible because of the confining lateral boundaries which did not allow deformation to result from application of the electric field (no electrostriction) but, instead, generated a residual stress. In the present problem the boundary conditions are different and application of an electric field (radial or axial) will cause the material to deform. For this reason we consider the electric field $\mathbf{E}$ in the deformed configuration and construct the independent variable $\mathbf{E}_{l}$ from it via the formula $\mathbf{E}_{l}=\mathbf{F}^{T} \mathbf{E}$, with $\mathbf{D}$ then being given by the constitutive equation (28).

\subsubsection{Electric field components}

As for the azimuthal shear problem there can be no azimuthal electric field. Thus, we restrict attention to an electric field with radial and axial components $E_{r}$ and $E_{z}$, respectively. Because of the form of the deformation, it follows that $D_{\theta}=0$. Since the equations $\operatorname{div} \mathbf{D}=0$ and $\operatorname{curl} \mathbf{E}=\mathbf{0}$ must hold and there is dependence only on $r$ we deduce that

$$
r D_{r}=\text { constant }, \quad E_{z}=\text { constant }
$$


but no restrictions are placed on $D_{z}$ and $E_{r}$ by these equations.

In view of the form of the deformation, from the connection $\mathbf{E}_{l}=\mathbf{F}^{T} \mathbf{E}$ we obtain the components

$$
E_{l R}=\lambda^{-1} \lambda_{z}^{-1} E_{r}, \quad E_{l Z}=\lambda_{z} E_{z} .
$$

The invariants $I_{4}, I_{5}, I_{6}$ given by (26) now specialize to

$$
I_{4}=E_{l R}^{2}+E_{l Z}^{2}, \quad I_{5}=\lambda^{2} \lambda_{z}^{2} E_{l R}^{2}+\lambda_{z}^{-2} E_{l Z}^{2}, \quad I_{6}=\lambda^{4} \lambda_{z}^{4} E_{l R}^{2}+\lambda_{z}^{-4} E_{l Z}^{2},
$$

the components of the magnetic induction vector $\mathbf{D}$ are

$$
\begin{aligned}
& D_{r}=-2\left(\Omega_{4} \lambda^{-2} \lambda_{z}^{-2}+\Omega_{5}+\Omega_{6} \lambda^{2} \lambda_{z}^{2}\right) E_{r}, \\
& D_{z}=-2\left(\Omega_{4} \lambda_{z}^{2}+\Omega_{5}+\Omega_{6} \lambda_{z}^{-2}\right) E_{z},
\end{aligned}
$$

and the corresponding components of the polarization are given by equation $(20)_{1}$. Note that, in view of $(83)_{1}$, equation (86) can be regarded as determining $E_{r}$ when the form of the function $\Omega$ is given. Thus, no restriction on $\Omega$ analogous to that on $\Omega^{*}$ in the azimuthal shear problem, i.e. (53), arises from this correlation.

\subsubsection{Stress components and equilibrium equation}

From equation (27) we obtain the non-zero components of the stress $\boldsymbol{\tau}$ as

$$
\begin{aligned}
\tau_{r r} & =-p+2 \lambda^{-2} \lambda_{z}^{-2}\left[\Omega_{1}+\Omega_{2}\left(\lambda^{2}+\lambda_{z}^{2}\right)\right]-2\left(\Omega_{5}+2 \Omega_{6} \lambda^{2} \lambda_{z}^{2}\right) E_{r}^{2}, \\
\tau_{\theta \theta} & =-p+2 \lambda^{2}\left[\Omega_{1}+\Omega_{2}\left(\lambda^{-2} \lambda_{z}^{-2}+\lambda_{z}^{2}\right)\right] \\
\tau_{z z} & =-p+2 \lambda_{z}^{2}\left[\Omega_{1}+\Omega_{2}\left(\lambda^{-2} \lambda_{z}^{-2}+\lambda^{2}\right)\right]-2\left(\Omega_{5}+2 \Omega_{6} \lambda_{z}^{-2}\right) E_{z}^{2}, \\
\tau_{r z} & =-2 E_{r} E_{z}\left[\Omega_{5}+\Omega_{6}\left(\lambda^{2} \lambda_{z}^{2}+\lambda_{z}^{-2}\right)\right] .
\end{aligned}
$$

The equilibrium equation $\operatorname{div} \boldsymbol{\tau}=\mathbf{0}$ reduces to the two component equations

$$
\frac{\mathrm{d}}{\mathrm{d} r}\left(r \tau_{r r}\right)=\tau_{\theta \theta}, \quad \frac{\mathrm{d}}{\mathrm{d} r}\left(r \tau_{r z}\right)=0 .
$$

The latter equation integrates to give $r \tau_{r z}=$ constant, which must be consistent with the expression (91). This point will be considered in Section 4.2.5.

Suppose the exterior of the body is a vacuum, for example. Then, by specializing equation (14), the Maxwell stress has non-zero components given by

$$
-\tau_{m r r}=\tau_{m z z}=\tau_{m \theta \theta}+\varepsilon_{0} E_{z}^{2}=-\frac{1}{2} \varepsilon_{0}\left(E_{r}^{2}-E_{z}^{2}\right), \quad \tau_{m r z}=\varepsilon_{0} E_{r} E_{z} .
$$

The loads associated with these stress components need to be accounted for in considering the continuity of traction on the boundaries $r=a, b$.

In the following we consider separately an axial and then a radial electric field, followed by a brief examination of their combined effect. 


\subsubsection{Axial electric field}

We now specialize to the case $E_{r}=0$, so that the invariants (85) reduce to

$$
I_{4}=E_{l Z}^{2}=\lambda_{z}^{2} E_{z}^{2}, \quad I_{5}=\lambda_{z}^{-2} I_{4}, \quad I_{6}=\lambda_{z}^{-4} I_{4},
$$

while $I_{1}$ and $I_{2}$ are unchanged and given by (82).

Since the invariants $I_{1}, I_{2}, I_{4}, I_{5}, I_{6}$ depend only on the three independent quantities $\lambda, \lambda_{z}, I_{4}$, it is convenient, in parallel with the definition of $\omega^{*}$ in (60), to define a reduced energy function $\omega$ in the form

$$
\omega\left(\lambda, \lambda_{z}, I_{4}\right)=\Omega\left(I_{1}, I_{2}, I_{4}, I_{5}, I_{6}\right)
$$

in which the right-hand side is evaluated for (82) and (94). This allows the stress differences to be written in the simple forms

$$
\tau_{\theta \theta}-\tau_{r r}=\lambda \omega_{\lambda}, \quad \tau_{z z}-\tau_{r r}=\lambda_{z} \omega_{\lambda_{z}},
$$

where $\omega_{\lambda}=\partial \omega / \partial \lambda$ and $\omega_{\lambda_{z}}=\partial \omega / \partial \lambda_{z}$. In terms of $\omega$ equation (87) simplifies to

$$
D_{z}=-2 \lambda_{z}^{2} \omega_{4} E_{z}
$$

where $\omega_{4}=\partial \omega / \partial I_{4}$. The corresponding polarization component is obtained from equation $(20)_{1}$ and is given by

$$
P_{z}=D_{z}-\varepsilon_{0} E_{z}=-\left(2 \omega_{4} \lambda_{z}^{2}+\varepsilon_{0}\right) E_{z} .
$$

Since $E_{r}=E_{\theta}=0$ it follows that $D_{r}=D_{\theta}=0$ and $P_{r}=P_{\theta}=0$.

The equilibrium equation $(92)_{1}$ can now be written

$$
r \frac{\mathrm{d} \tau_{r r}}{\mathrm{~d} r}=\lambda \omega_{\lambda}
$$

while, since $E_{r}=0$ and hence, by $(91), \tau_{r z}=0$, equation $(92)_{2}$ is satisfied identically.

The Maxwell stress components (93) reduce to

$$
\tau_{m r r}=\tau_{m \theta \theta}=-\frac{1}{2} \varepsilon_{0} E_{z}^{2}=-\tau_{m z z} .
$$

Now, according to the continuity condition $(12)_{2}, E_{z}$ must be continuous across the boundaries $r=a, b$, and since it is independent of $r$ within the material it is spatially uniform for all $r$. Note that $\tau_{m r r}$ contributes to the radial traction on the boundaries $r=a$ and $r=b$ and the axial stress $\tau_{m z z}$ contributes to the resulting axial load on any cross-section of the tube. 
Let the tube be subjected to a pressure $P$ on the inner surface $r=a$ with no mechanical loads applied to the outside surface $r=b$. The two boundary conditions, including the radial Maxwell stress component $\tau_{m r r}$, are

$$
\tau_{r r}=-P-\frac{1}{2} \varepsilon_{0} E_{z}^{2} \quad \text { on } \quad r=a, \quad \tau_{r r}=-\frac{1}{2} \varepsilon_{0} E_{z}^{2} \quad \text { on } \quad r=b .
$$

Integration of equation (99) and use of the boundary conditions (101) leads to

$$
P=\int_{a}^{b} \lambda \omega_{\lambda} \frac{\mathrm{d} r}{r}
$$

Since, from (81), we have $b^{2}=a^{2}+\lambda_{z}^{-1}\left(B^{2}-A^{2}\right)$, equation (102) provides a relationship between the pressure $P$ and the inner deformed radius $a$ when $\lambda_{z}$ is known. Note that this formula does not involve the Maxwell stress since $\tau_{m r r}$ has the same value on each boundary. Thus, (102) has precisely the same form as for a purely elastic material (see, for example, Haughton and Ogden [7]) except that $\omega$ here depends on $I_{4}$.

The resultant axial load $N$ on any cross-section of the tube is calculated from

$$
N=2 \pi \int_{a}^{b} \tau_{z z} r \mathrm{~d} r .
$$

Use of the expressions for the stress differences in (96), the equilibrium equation $(92)_{1}$, the boundary conditions (101) and the expression in (100) for $\tau_{m z z}$ gives, for the axial load $N$,

$$
N=\pi \int_{a}^{b}\left(2 \lambda_{z} \omega_{\lambda_{z}}-\lambda \omega_{\lambda}\right) r \mathrm{~d} r+\pi a^{2} P-\frac{1}{2} \pi\left(b^{2}-a^{2}\right) \varepsilon_{0} E_{z}^{2} .
$$

For the corresponding expression in the purely elastic case, we refer to Ogden [12], for example.

Suppose now we examine the influence of the electric field alone with no mechanical tractions applied, i.e. we set $P=0$. Consider the integrand in (102), which, by (88), (89) and (96) $)_{1}$, gives

$$
\lambda \omega_{\lambda}=2 \lambda^{-2} \lambda_{z}^{-2}\left(\Omega_{1}+\lambda_{z}^{2} \Omega_{2}\right)\left(\lambda^{4} \lambda_{z}^{2}-1\right) .
$$

For an elastic material the term $\Omega_{1}+\lambda_{z}^{2} \Omega_{2}$ is usually taken to be positive (this follows from the Baker-Ericksen inequalities, for example, and the term is also closely related to the shear modulus of the material; for details see, for example, Truesdell and Noll [15]). We therefore adopt this condition also in the present context. Now, from equation $(81)_{1}$ and the definition of $\lambda(=r / R)$, we obtain

$$
\left(\lambda^{2} \lambda_{z}-1\right) R^{2}=\left(\lambda_{a}^{2} \lambda_{z}-1\right) A^{2}
$$


where $\lambda_{a}=a / A$. This shows that $\lambda^{2} \lambda_{z}-1$ is either positive, negative or zero throughout the range $A \leq R \leq B$, and hence the integral (102) cannot vanish unless $\lambda^{2} \lambda_{z}=1$ for $A \leq R \leq B$. The deformation must therefore be homogeneous and the radial stress must be uniform and equal to the Maxwell stress $-\varepsilon_{0} E_{z}^{2} / 2$. The resulting value of the axial load $N$ is

$$
N=\pi\left(b^{2}-a^{2}\right)\left(\lambda_{z} \omega_{\lambda_{z}}-\frac{1}{2} \varepsilon_{0} E_{z}^{2}\right) .
$$

From equations (88), (90) and (96), on use of the connection $\lambda^{2} \lambda_{z}=1$, we obtain

$$
\lambda_{z} \omega_{\lambda_{z}}=2 \lambda_{z}^{-1}\left(\Omega_{1}+\lambda_{z}^{-1} \Omega_{2}\right)\left(\lambda_{z}^{3}-1\right)-2\left(\Omega_{5}+2 \lambda_{z}^{-2} \Omega_{6}\right) E_{z}^{2},
$$

evaluated for $\lambda^{2} \lambda_{z}=1$.

One possible solution of interest arises when $\lambda=\lambda_{z}=1$. Then, on use of equation (108), equation (107) reduces to

$$
N=-2 \pi\left(b^{2}-a^{2}\right)\left(\Omega_{5}+2 \Omega_{6}+\frac{1}{4} \varepsilon_{0}\right) E_{z}^{2} .
$$

This is the axial load required to maintain the undeformed configuration in the presence of the applied axial electric field. If the sign of the term $\Omega_{5}+2 \Omega_{6}+\frac{1}{4} \varepsilon_{0}$ is positive (negative) then $N$ is negative (positive) and the electric field tends to lengthen (shorten) the tube.

\subsubsection{Radial electric field}

We now take $E_{z}=0$ and consider a radial field with component $E_{r}$. The invariants (85) are then reduced to

$$
I_{4}=E_{l R}^{2}=\lambda^{-2} \lambda_{z}^{-2} E_{r}^{2}, \quad I_{5}=\lambda^{2} \lambda_{z}^{2} I_{4}, \quad I_{6}=\lambda^{4} \lambda_{z}^{4} I_{4} .
$$

As in Section 4.2.3 we define the reduced energy function $\omega$ according to (95), but now with $I_{4}, I_{5}, I_{6}$ defined by (110). The expressions for the principal stress differences in the material have the forms given by equation (96) and are not be repeated here, and again $\tau_{r z}=0$. From equation (86) we obtain

$$
D_{r}=-2 \lambda^{-2} \lambda_{z}^{-2} \omega_{4} E_{r},
$$

and the corresponding polarization is

$$
P_{r}=D_{r}-\varepsilon_{0} E_{r}=-\left(2 \omega_{4} \lambda^{-2} \lambda_{z}^{-2}+\varepsilon_{0}\right) E_{r} .
$$


Outside the tube, the non-zero Maxwell stress components are given by

$$
\tau_{m \theta \theta}=\tau_{m z z}=-\frac{1}{2} \varepsilon_{0} E_{r}^{2}=-\tau_{m r r}
$$

In the present situation, since $E_{r}$ depends on $r$, the Maxwell stress influences the pressure-radius relation, which, on integration of (99) again and use of the expression for $\tau_{m r r}$ in (113), takes the form

$$
P+\tau_{m}(b)-\tau_{m}(a)=\int_{a}^{b} \lambda \omega_{\lambda} \frac{\mathrm{d} r}{r}
$$

where $\tau_{m}(r)=\varepsilon_{0} E_{r}^{2} / 2$.

The axial load $N$ is obtained similarly to equation (104) and is given by

$$
N=\pi \int_{a}^{b}\left(2 \lambda_{z} \omega_{\lambda z}-\lambda \omega_{\lambda}\right) r \mathrm{~d} r+\pi a^{2} P+\pi\left[b^{2} \tau_{m}(b)-a^{2} \tau_{m}(a)\right]
$$

However, the latter term in (115) vanishes and the Maxwell stress does not affect the axial load, as we now show.

First, we recall from (83) that $r D_{r}=c$, where $c$ is a constant. Also, by the continuity condition (12), specialized to the present situation, we see that $D_{r}$ is continuous across the surfaces $r=a, b$. Moreover, since $\mathbf{D}=\varepsilon_{0} \mathbf{E}$ outside the material, it follows that the values of $E_{r}$ required in the Maxwell stress expressions (113) are $c / a \varepsilon_{0}$ and $c / b \varepsilon_{0}$ on $r=a$ and $r=b$, respectively. Hence, $b^{2} \tau_{m}(b)=a^{2} \tau_{m}(a)\left(=c^{2} / 2 \varepsilon_{0}\right)$.

Now, with reference to (114), we calculate

$$
\tau_{m}(b)-\tau_{m}(a)=-\frac{\left(b^{2}-a^{2}\right) c^{2}}{2 \varepsilon_{0} a^{2} b^{2}}
$$

which is negative. Hence, the effect of a radial electric field is to counteract the effect of an inflating pressure $(P>0)$.

For this problem we have

$$
\begin{gathered}
\lambda \omega_{\lambda}=2 \lambda^{-2} \lambda_{z}^{-2}\left(\Omega_{1}+\lambda_{z}^{2} \Omega_{2}\right)\left(\lambda^{4} \lambda_{z}^{2}-1\right)+2\left(\Omega_{5}+2 \lambda^{2} \lambda_{z}^{2} \Omega_{6}\right) E_{r}^{2}, \\
\lambda_{z} \omega_{\lambda_{z}}=2 \lambda^{-2} \lambda_{z}^{-2}\left(\Omega_{1}+\lambda^{2} \Omega_{2}\right)\left(\lambda^{2} \lambda_{z}^{4}-1\right) .
\end{gathered}
$$

If $P=0$ and the electric field is applied in such a way that the reference configuration $\lambda=\lambda_{z}=1$ is maintained then

$$
\tau_{m}(b)-\tau_{m}(a)=2 \int_{a}^{b}\left(\Omega_{5}+2 \Omega_{6}\right) E_{r}^{2} \frac{\mathrm{d} r}{r},
$$


which must be negative. The inequality $\Omega_{5}+2 \Omega_{6}<0$ is consistent with this requirement, and if it holds then

$$
N=-2 \pi \int_{a}^{b}\left(\Omega_{5}+2 \Omega_{6}\right) E_{r}^{2} r \mathrm{~d} r
$$

is positive, i.e. a tensile axial load is required to prevent the tube shortening under the influence of the electric field.

By contrast, if we consider an energy function of the form

$$
\Omega\left(I_{1}, I_{4}\right)=\frac{1}{2} \mu\left(I_{4}\right)\left(I_{1}-3\right)+\nu\left(I_{4}\right) \longrightarrow \omega\left(\lambda, \lambda_{z}, I_{4}\right),
$$

where $\mu\left(I_{4}\right)$ and $\nu\left(I_{4}\right)$ are functions of $I_{4}$ analogous to $\mu^{*}\left(K_{4}\right)$ and $\nu^{*}\left(K_{4}\right)$ in (71) with $k=1$, then, for $\lambda_{z}$ fixed at 1 , we obtain, for $P=0$,

$$
\tau_{m}(b)-\tau_{m}(a)=\int_{a}^{b} \mu\left(I_{4}\right)\left(\lambda^{2}-\lambda^{-2}\right) \frac{\mathrm{d} r}{r} .
$$

For this to be negative (assuming $\mu\left(I_{4}\right)>0$ ) we require $\lambda<1$, and then

$$
N=-\pi \int_{a}^{b} \mu\left(I_{4}\right)\left(\lambda-\lambda^{-1}\right)^{2} r \mathrm{~d} r
$$

is also negative, thus giving the opposite characteristics compared with the previous example. The electric field tends to lengthen the tube. Clearly, the issue of the choice of energy function is critical here and requires more investigation in the light of experimental data, of which there is relatively little available for the types of material envisaged.

\subsubsection{Combined axial and radial electric fields}

When $E_{r} \neq 0$ and $E_{z} \neq 0$ the stress component $\tau_{r z}$ given by (91) is in general non-zero. When it is combined with the solution of equation $(92)_{2}$, we obtain

$$
\tau_{r z}=-2\left[\Omega_{5}+\Omega_{6}\left(\lambda^{2} \lambda_{z}^{2}+\lambda_{z}^{-2}\right)\right] E_{r} E_{z}=\frac{c_{\tau}}{r},
$$

where $c_{\tau}$ is a constant. Similarly, for the radial component $D_{r}$ we have, from (86) combined with (83),

$$
D_{r}=-2\left(\Omega_{4} \lambda^{-2} \lambda_{z}^{-2}+\Omega_{5}+\Omega_{6} \lambda^{2} \lambda_{z}^{2}\right) E_{r}=\frac{c_{d}}{r},
$$

where $c_{d}$ is a constant. 
The stress $\tau_{r z}$ must match the Maxwell stress $\tau_{m r z}$ given by (93) on $r=a$ and $r=b$ if there is no applied mechanical shear traction. Now $\tau_{m r z}=$ $\varepsilon_{0} E_{r} E_{z}$, which, since $\mathbf{D}=\varepsilon_{0} \mathbf{E}$ outside the material, can also be written $D_{r} E_{z}$. But $D_{r}$ is continuous across any surface $r=$ constant, so that, on $r=a$, for example, we have $\tau_{m r z}=c_{d} E_{z} / a$, while $\tau_{r z}=c_{\tau} / a$. Since these must be equal, we have the connection

$$
c_{\tau}=c_{d} E_{z}
$$

(recall that $E_{z}$ is a constant).

Using this latter connection and combining (124) and (125) we obtain, after a little rearrangement, the restriction

$$
\Omega_{4}=\lambda^{2} \Omega_{6}
$$

on $\Omega$. Thus, only energy functions satisfying this condition can support the two-component electric field for the considered deformation.

It is interesting to write (127) in an alternative way. For this purpose we note the connection

$$
I_{6}=\left(\lambda^{2} \lambda_{z}^{2}+\lambda_{z}^{-2}\right) I_{5}-\lambda^{2} I_{4}
$$

which can be obtained from (84) and (85). This means that since there are only two components of the electric field only two of the three invariants $I_{4}, I_{5}, I_{6}$ are independent, which prompts the introduction of another reduced form of $\Omega$. We denote this by $\hat{\Omega}$, which is a function of $I_{1}, I_{2}, I_{4}, I_{5}$ defined by

$$
\hat{\Omega}\left(I_{1}, I_{2}, I_{4}, I_{5}\right)=\Omega\left(I_{1}, I_{2}, I_{4}, I_{5}, I_{6}\right),
$$

with $I_{6}$ on the right-hand side replaced by the expression in (128). A simple calculation shows that $\hat{\Omega}_{4}=\Omega_{4}-\lambda^{2} \Omega_{6}$ and equation (127) therefore becomes

$$
\hat{\Omega}_{4}=0,
$$

which is satisfied for any $\hat{\Omega}$ of the form $\hat{\Omega}=\hat{\Omega}\left(I_{1}, I_{2}, I_{5}\right)$.

\section{$5 \quad$ Inflation of a spherical shell}

\section{$5.1 \quad$ Kinematics}

Consider a thick-walled spherical shell for which the reference configuration $\mathcal{B}_{0}$ is described in terms of spherical polar coordinates $(R, \Theta, \Phi)$ by

$$
A \leq R \leq B, \quad 0 \leq \Theta \leq \pi, \quad 0 \leq \Phi \leq 2 \pi,
$$


where $A$ and $B$ are the inner and outer radii of the shell. The shell is now inflated (or deflated) radially and symmetrically. Since we are considering an incompressible material, the deformation can be described in the standard form

$$
r^{3}=R^{3}+a^{3}-A^{3}, \quad \theta=\Theta, \quad \phi=\Phi,
$$

with $a \leq r \leq b$, where $(r, \theta, \phi)$ are spherical polar coordinates in the deformed configuration and $a$ and $b$ the inner and outer radii of the deformed shell.

The deformation gradient with respect to the spherical polar coordinate axes is diagonal and the associated principal stretches are given by

$$
\lambda_{1}=\lambda^{-2}, \quad \lambda_{2}=\lambda_{3}=\lambda=\frac{r}{R},
$$

$\lambda$ being the (equibiaxial) principal stretch locally in the plane normal to the radial direction. In terms of $\lambda$ the associated principal invariants have the forms

$$
I_{1}=2 \lambda^{2}+\lambda^{-4}, \quad I_{2}=2 \lambda^{-2}+\lambda^{4} .
$$

\subsection{The electric and stress field components}

Because of the spherical symmetry we consider only a radial electric field and electric induction, with components $E_{r}$ and $D_{r}$, respectively, which depend only on $r$. The equation curl $\mathbf{E}$ is then satisfied identically, and $\operatorname{div} \mathbf{D}=0$ leads to

$$
r^{2} D_{r}=c,
$$

where again $c$ is a constant.

We work now in terms of the formulation based on use of $\mathbf{D}_{l}=\mathbf{F}^{-1} \mathbf{D}$ as the independent electric variable, which has just the single component $D_{l R}=\lambda^{2} D_{r}$. The associated invariants are then

$$
K_{4}=D_{l R}^{2}, \quad K_{5}=\lambda^{-4} K_{4}, \quad K_{6}=\lambda^{-8} K_{4} .
$$

The (isotropic) energy function $\Omega^{*}$ is regarded as a function of $I_{1}, I_{2}, K_{4}, K_{5}, K_{6}$.

The electric field component $E_{r}$ is obtained from equation (33) as

$$
E_{r}=2\left(\Omega_{4}^{*} \lambda^{4}+\Omega_{5}^{*}+\Omega_{6}^{*} \lambda^{-4}\right) D_{r}
$$

and the corresponding radial polarization is given by

$$
P_{r}=D_{r}-\varepsilon_{0} E_{r}=\left[1-2 \varepsilon_{0}\left(\Omega_{4}^{*} \lambda^{4}+\Omega_{5}^{*}+\Omega_{6}^{*} \lambda^{-4}\right)\right] D_{r} .
$$


From equation (32) the total stress tensor has components

$$
\begin{aligned}
\tau_{r r} & =-p+2\left[\Omega_{1}^{*} \lambda^{-4}+2 \Omega_{2}^{*} \lambda^{-2}+\left(\Omega_{5}^{*} \lambda^{-4}+2 \Omega_{6}^{*} \lambda^{-8}\right) K_{4}\right] \\
\tau_{\theta \theta} & =\tau_{\phi \phi}=-p+2\left[\Omega_{1}^{*} \lambda^{2}+\Omega_{2}^{*}\left(\lambda^{4}+\lambda^{-2}\right)\right]
\end{aligned}
$$

and the equilibrium equation $(40)_{1}$ reduces, for the spherical symmetric deformation considered, to the radial component equation

$$
\frac{\mathrm{d} \tau_{r r}}{\mathrm{~d} r}=\frac{2}{r}\left(\tau_{\theta \theta}-\tau_{r r}\right)
$$

For the spherically-symmetric deformation of the spherical shell subject to a radial electric field all invariants can be expressed in terms of two independent quantities, namely the stretch $\lambda$ and the invariant $K_{4}$. It is therefore convenient to introduce again a reduced energy function $\omega^{*}$, this time defined by

$$
\omega^{*}\left(\lambda, K_{4}\right)=\Omega^{*}\left(I_{1}, I_{2}, K_{4}, K_{5}, K_{6}\right),
$$

with $I_{1}$ and $I_{2}$ given by $(134)$ and $K_{4}, K_{5}, K_{6}$ by (136). The radial electric field (137) and the polarization (138) are then given simply by

$$
E_{r}=2 \lambda^{4} \omega_{4}^{*} D_{r}, \quad P_{r}=\left(1-2 \varepsilon_{0} \lambda^{4} \omega_{4}^{*}\right) D_{r},
$$

where $\omega_{4}^{*}=\partial \omega^{*} / \partial K_{4}$, and the stress difference via

$$
2\left(\tau_{\theta \theta}-\tau_{r r}\right)=\lambda \omega_{\lambda}^{*}
$$

where $\omega_{\lambda}^{*}=\partial \omega^{*} / \partial \lambda$. The equilibrium equation (141) can now be written

$$
r \frac{\mathrm{d} \tau_{r r}}{\mathrm{~d} r}=\lambda \omega_{\lambda}^{*}
$$

Outside the material, assumed to be vacuum, the electric field is given by $E_{r}=\varepsilon_{0}^{-1} D_{r}$ and, by (14), the radial component of the Maxwell stress is obtained as

$$
\tau_{m r r}=\frac{1}{2} \varepsilon_{0}^{-1} D_{r}^{2} \equiv \tau_{m}(r),
$$

wherein the notation $\tau_{m}(r)$ is defined.

The boundary $r=b$ is assumed to be free of mechanical traction and on the inner boundary $r=a$ a pressure $P$ is applied. On accounting for the Maxwell stress, it follows that the boundary conditions are

$$
\tau_{r r}=-P+\tau_{m}(a) \quad \text { on } \quad r=a, \quad \tau_{r r}=\tau_{m}(b) \quad \text { on } \quad r=b .
$$


Integration of equation (145) and use of the boundary conditions (147) yields

$$
P+\tau_{m}(b)-\tau_{m}(a)=\int_{a}^{b} \lambda \omega_{\lambda}^{*} \frac{\mathrm{d} r}{r} .
$$

For details of the analysis of the inflation of an elastic spherical shell and its stability we refer to, for example, Haughton and Ogden [6] and Ogden [12].

From (135) and (146) we obtain

$$
\tau_{m}(b)-\tau_{m}(a)=-\frac{\left(b^{4}-a^{4}\right) c_{d}^{2}}{2 \varepsilon_{0} a^{4} b^{4}},
$$

which is negative. Hence, similarly to the corresponding problem of a tube considered in Section 4.2.4, the radial field counteracts the effect of an inflating pressure.

From (139), (140) and (144) it follows that

$$
\lambda \omega_{\lambda}^{*}=4 \lambda^{-4}\left[\left(\Omega_{1}^{*}+\lambda^{2} \Omega_{2}^{*}\right)\left(\lambda^{6}-1\right)-\left(\Omega_{5}^{*}+2 \lambda^{-4} \Omega_{6}^{*}\right) K_{4}\right] .
$$

The reference configuration $\lambda=1$ can be maintained by the combined action of pressure and the electric field provided

$$
P+\tau_{m}(b)-\tau_{m}(a)=-4 \int_{a}^{b}\left(\Omega_{5}^{*}+2 \Omega_{6}^{*}\right) K_{4} \frac{\mathrm{d} r}{r} .
$$

More particularly, if $P=0$ then the right-hand side of (151) must be negative, which suggests that it is reasonable to adopt the inequality $\Omega_{5}^{*}+2 \Omega_{6}^{*}>0$. Note, however, that this does not hold for restricted models of the form (71) or for $\Omega^{*}=\Omega^{*}\left(I_{1}, I_{2}, K_{4}\right)$.

\section{Conclusions}

In this paper we have summarized the equations for a theory of nonlinear electroelasticity following the formulation of Dorfmann and Ogden [4] and then applied them in the case of an incompressible isotropic material to three problems that illustrate the effects of electroelastic interactions in electrosensitive materials that are capable of large deformations.

Two alternative constitutive formulations, based on different choices of independent electric variable, have been used. The selection of the electric field or the electric induction as the independent variable is an important consideration. For certain combinations of deformations and electric fields one choice of the independent variable may lead to restrictions on the admissible class of constitutive laws while the other does not, as has been discussed 
by Bustamante and Ogden [2] and, in the context of magnetoelasticity, by Dorfmann and Ogden [3].

A key issue is the selection of realistic forms of energy function $\Omega$ (or $\Omega^{*}$ ) to model the response of large deformation electro-sensitive materials, such as ES elastomers. Here, in Section 4.1.3, in the reduced form of $\omega^{*}$ given by (65), we have introduced a prototype model, but further exploration of the efficacy of specific models is needed from several perspectives. These include mathematical aspects concerned with the properties of the governing equations, the evaluation of stability inequalities, such as strong ellipticity, and the assessment of large deformation material response against a body of experimental data that catalogues the dependence of the mechanical response on the electric field strength and direction for specific geometries. There is a pressing need for comprehensive sets of data since data of the required kind are not currently available.

\section{References}

[1] Y. Bar-Cohen, Electro-active polymers: current capabilities and challenges. In Y. Bar-Cohen (ed.), Proceedings of the 4th Electroactive Polymer Actuators and Devices (EAPAD) Conference, 9th Smart Structures and Materials Symposium, Vol. 4695, San Diego (2002), pp. 1-7.

[2] R. Bustamante and R. W. Ogden, Universal relations for nonlinear electroelastic solids. Acta Mechanica, in press (2005).

[3] A. Dorfmann and R. W. Ogden, Nonlinear magnetoelastic deformations. Q. J. Mech. Appl. Math. 57 (2004) 599-622.

[4] A. Dorfmann and R. W. Ogden, Nonlinear electroelasticity. Acta Mechanica 174 (2005) 167-183.

[5] A. C. Eringen and G. A. Maugin, Electrodynamics of Continua I. Springer, New York (1990).

[6] D. M. Haughton and R. W. Ogden, On the incremental equations in non-linear elasticity - II. Bifurcation of pressurized spherical shells. J. Mech. Phys. Solids 26 (1978) 111-138.

[7] D. M. Haughton and R. W. Ogden, Bifurcation of inflated circular cylinders of elastic material under axial loading - II. Exact theory for thickwalled tubes. J. Mech. Phys. Solids 27 (1979) 489-512. 
[8] K. Hutter and A. A. F. van de Ven, Field Matter Interactions in Thermoelastic Solids. Lecture Notes in Physics, Vol. 88. Springer, Berlin (1978).

[9] X. Jiang and R. W. Ogden, On azimuthal shear of a circular cylindrical tube of compressible elastic material. Q. J. Mech. Appl. Math. 51 (1998) $143-158$.

[10] A. Kovetz, Electromagnetic Theory. Oxford University Press (2000).

[11] G. A. Maugin, Continuum Mechanics of Electromagnetic Solids. North Holland, Amsterdam (1988).

[12] R. W. Ogden, Non-linear Elastic Deformations. Dover, New York (1997)

[13] R. W. Ogden, Elements of the theory of finite elasticity. In Y. B. Fu and R. W. Ogden (eds), Nonlinear Elasticity: Theory and Applications. Cambridge University Press (2001), pp. 1-57.

[14] A. J. M. Spencer, Theory of Invariants. In A. C. Eringen (ed.), Continuum Physics, Vol. 1. Academic Press, New York (1971), pp. 239-353.

[15] C. Truesdell and W. Noll, The Nonlinear Field Theories of Mechanics. In S. Flügge (ed.), Handbuch der Physik, Vol. III/3. Springer, Berlin (1965).

[16] C. Truesdell and R. Toupin, The Classical Field Theories. In S. Flügge (ed.), Handbuch der Physik, Vol. III/1. Springer, Berlin (1960). 